\title{
Spatial and Temporal Variability of Phytoplankton at Hwadang-ri, Goseng-gun
}

\author{
Man Ki Kang ${ }^{1}$ and Man Kyu Huh ${ }^{2 *}$ \\ ${ }^{1}$ Department of Data Information Science, College of Natural Sciences \& Human Ecology, Dongeui University, Busan 614-714, Korea \\ ${ }^{2}$ Department of Molecular Biology, College of Natural Sciences \& Human Ecology, Dongeui University, Busan 614-714, Korea
}

Received March 4, 2014 /Revised May 2, 2014 /Accepted May 7, 2014

\begin{abstract}
This study describe seasonal patterns in the variation of phytoplankton frequency in the water surface and basal layers and their spatial distributions at seven stations in Hwadang-ri, Goseng-gun in 2013. The phytoplankton community at Hwadang-ri was very diverse, with 60 taxa identified, representing three classes. Diatoms (Bacillariophyceae) exhibited the greatest diversity, with 41 taxa identified. These were followed by the dinoflagellates Dinophyceae, Cryptophyceae, and Eugenophyceae, with 16 taxa, two taxa, and one taxon, respectively. Water surfaces were shown with the relative individual density or abundance across areas. Except in January, Shannon-Weaver indices of diversity of the water surface layer were lower than those of the basal layer. In addition, evenness indices of the basal layer were higher than those of the water surface layer, except in January. For the community as a whole, the values of ß-diversity were low for the seven stations: 1.125 for the water surface layer and 1.481 for the basal layer. Seasonal values for ß-diversity were similar at the seven stations: 1.725 for the water surface layer and 1.347 for the basal layer. The phytoplankton community showed high taxonomic homogeneity in all four seasons, in addition to similar trends in seasonal development at depths in the same stations. However, the size distribution of the abundance and biomass showed a statistically significant west-east difference.
\end{abstract}

Key words : Hwadang-ri, phytoplankton, size - frequency distribution, spatial patterns, ß-diversity

\section{Introduction}

Phytoplankton is photosynthesizing microscopic organisms and the autotrophic components of the plankton community. They inhabit most the upper sunlit layer of almost all oceans and bodies of fresh water. Despite their infinitely small size in comparison to other marine organisms, these tiny creatures occupy an immensely important ecological niche. They are agents for very important primary production of earth. By the action of the sun's rays on chlorophyll (light absorbing pigments found within the phytoplankton cell) these plants produce carbohydrates, proteins, fats, and oxygen [1]. These products in turn are consumed directly or indirectly by all other marine life forms from zooplankton to fishes. Thus, phytoplankton has a vastly significant role to play not only in the marine food web of which they are part of, but also on a more global scale [3].

\footnotetext{
*Corresponding author

Tel : +82-51-890-1521, Fax : +82-51-890-1529

E-mail : mkhuh@deu.ac.kr

This is an Open-Access article distributed under the terms of the Creative Commons Attribution Non-Commercial License (http://creativecommons.org/licenses/by-nc/3.0) which permits unrestricted non-commercial use, distribution, and reproduction in any medium, provided the original work is properly cited
}

Phytoplankton can be account for half of all photosynthetic activity on Earth [18]. Therefore, their significance extends far beyond the marine environment alone. In addition to produce the carbohydrates, phytoplankton take up dissolved carbon dioxide in the process of photosynthesis and then give off oxygen. The fish consume the carbon fixed by the plants, use the dissolved oxygen for respiration, and release carbon dioxide. They play key roles in supporting all other organisms in the marine environment, as well as in the regulation of the Earth's climate through the sequestration of carbon, oxygen production, and other related processes [2].

There are two kinds of ocean currents; surface currents which extend only a few feet below the surface and subsurface currents that run below the surface depths [12]. Factors affecting the depth of the euphotic zone are the incidental angle of sunlight, the clarity of the atmosphere, and the turbidity of the water.

The spatial and seasonal changes of marine algae are important because they can produce a variety of highly toxic compounds - marine biotoxins [13]. These compounds, some of which can be released to the surrounding water while others are retained in the phytoplankton, can enter the food web and accumulate in fish and shellfish [22]. In some cases higher in the food web, fish and shellfish can be affected 
by these potent compounds and made ill or even die. In virtually all cases, the marine biotoxins produced by these phytoplankton [8]. We surveyed the some examples of phytoplankton in the surface and subsurface at Hwadang-ri, Georyu-meon, Goseng-gun, Gyeongsangnam-do. Red tides usually occur along the south coasts near to this area in late summer and autumn [7]. Most red tides along the South Korea coast are caused by a group of phytoplankton known as dinoflagellates [8]. These single-celled organisms are able to swim short distances by means of two whip-like appendages called flagella.

Therefore, the present study aimed to examine the taxonomic structure of phytoplankton to provide preliminary information at Hwadang-ri which was characterized by tidal regimes ensuring high openness and low water turnover times at high tides. We describe more in details taxonomic composition of diatoms about spatial and temporal variability of phytoplankton.

\section{Materials and Methods}

\section{Sampling of phytoplankton}

Plankton samplings were conducted at seven stations at Hwadang-ri, Georyu-meon, Goseng-gun, Gyeongsangnamdo (Fig. 1). Sampling periods were from 28 January, 14 April, 03 August, and 27 October 2013. Two step samples from the surface layer $(1 \mathrm{~m}$ depth) to basal layer $(20 \mathrm{~m}$ depth) were collected by 5 liter Niskin bottles and preserved with acidified Lugol solution.

A water bottles sample contains all but the rarest organ- isms in the water mass sampled and includes the whole size spectrum from the largest entities, like diatom colonies to the smallest single cells [20]. These are ideal for quantitative phytoplankton collections as required quantities of water can be collected from the desired depth [17].

\section{Identification of phytoplankton}

Identification of diatoms in water samples is usually best done by using phase contrast optics, which reveal especially well lightly silicified structures, like delicate Chaetoceros setae, and also the organic chitin threads in Thallassiosiraceae [20].

It is essential to know which side of the diatoms cell is viewed [17]. Intact single cells with a short pervalvar axis tend to lie up under the coverslip (Coscinodiscus sp and Pleurosigma sp). Diatoms like Rhizosolenia with a pervalvar axis longer than the cell diameter or the apical axis turn girdle side upwards. Colony types like Chaetoceros, Fragilariopsis and Thalassiosira are normally seen in girdle view in a water mount. Diatoms like Thalassionema, Asterionellopsis and Pseudo-nitzschia show either valve or girdle side. Cylindrical and discoid diatoms are readily recognized by the general circular outlines in valves view. When the cells are viewed properly the next step is to look for special features like setae in Chaetoceraceae, shape of linking processes in Skelotonema and in unpreserved material, organic threads from the valve in Thallassiosiraceae.

Frustular elements cleaned of organic material may also be oriented in various ways in a permanent mount [20]. Flattened valves with a low mantle will usually be seen in valve view (Some Coscinodiscus spp., most Navicula spp.),
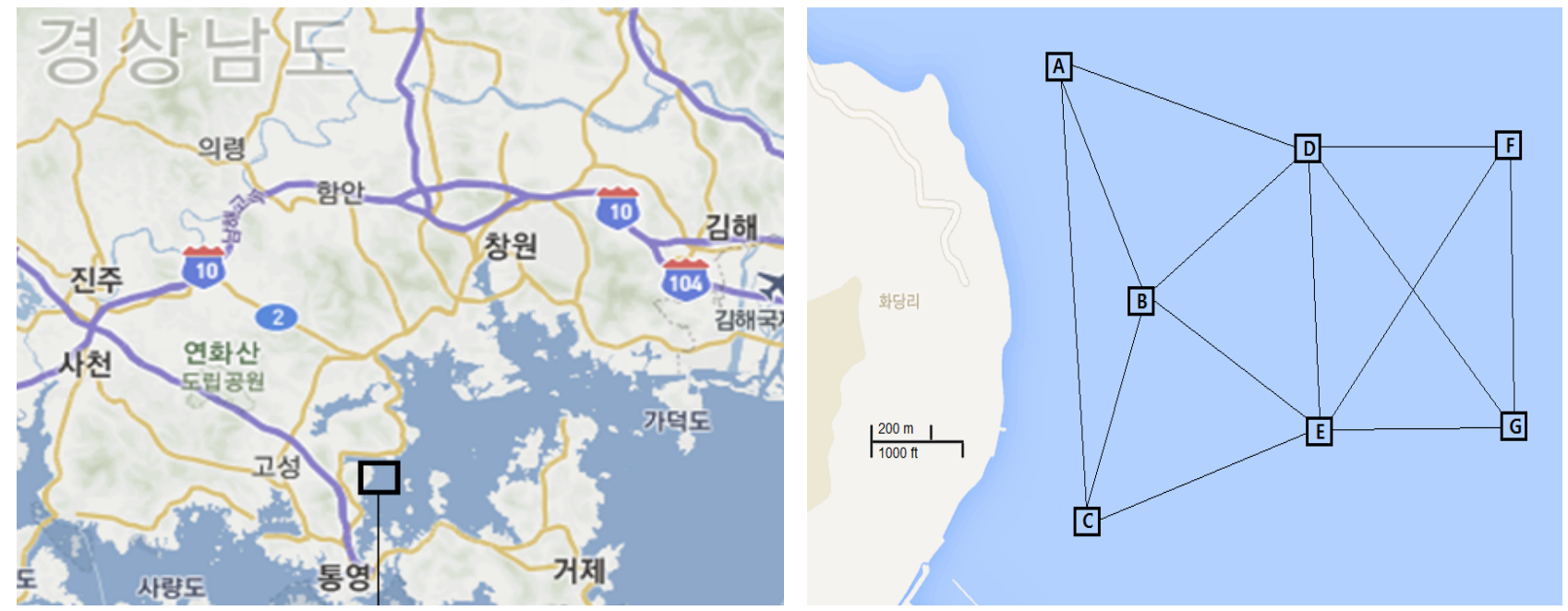

Fig. 1. The seven stations at Hwadang-ri, Georyu-meon, Goseng-gun. 
while valves with a high mantle and protuberances may appear in girdle view (Eucampia and Rhizosolenia). Lightly silicified bands shaped as those in Rhizosolenia and Stephanopyxis often lie with girdle side up.

\section{Cell counts}

The direct estimate of phytoplankton cell density as measures of standing crop was made by this method [17]. The enumeration of phytoplankton is done by various counting chambers, however, the most commonly used counting chamber is Sedgwick Rafter cell. The counting cell is filled with the plankton sample and placed on the mechanical stage of the microscope. Then the counting cell is left for about half-an-hour for proper sedimentation. The organisms are then counted from one corner of the counting cell to the other.

\section{Biotic indices}

Shannon - Weaver index of diversity [14, 15]: the formula for calculating the Shannon diversity index is

$\mathrm{H}^{\prime}=-\sum \not \dot{i}$ In $\not \dot{x}$

Where, $\mathrm{H}^{\prime}=$ Shannon index of diversity.

$p \dot{p}=$ the proportion of important value of the th species $(p \dot{i}=m \mathrm{i} / \mathrm{N}, r \dot{\mathrm{d}}$ is the important value index of th species and $\mathrm{N}$ is the important value index of all the species).

$$
\begin{aligned}
& \mathrm{R} 1=(\mathrm{S}-1) / \mathrm{In}(\mathrm{n}) \\
& \mathrm{R} 2=\mathrm{S} / \sqrt{ } \mathrm{n}
\end{aligned}
$$

The species richness of phytoplankton was calculated by using the method, Margalef's index of richness [10].

$$
\begin{aligned}
& N 1=e^{H^{\prime}} \\
& N 2=1 / \sum_{\mathrm{i}=1}^{s} p i^{2}
\end{aligned}
$$

Evenness indices (E1 E5) were calculated using important value index of species using Hill's methods [5].

\section{Spatial correlation coefficients and cluster analysis}

Cluster analysis was applied to generate dendrograms (group average method), based on the Jaccard distance matrixes among samples. Calculation of indices and cluster analysis were performed using Primer 6.1.9 software (Primer-E Ltd.). The correlation coefficient is calculated for estimates of the relationships between geographic distance and the phytoplankton community. Except where stated otherwise, statistical analyses were performed using the SPSS software (Release 21.0) [6].

\section{Results}

\section{Composition and biomass of species}

The phytoplankton community at Hwadang-ri on 2013 was identified with 60 taxa, representing three classes (Table 1). Diatoms (Bacillariophyceae) exhibited the greatest diversity with 41 taxa identified, followed by dinoflagellates (Dinophyceae, 16 taxa); Cryptophyceae with two taxa, and Eugenophyceae represented by a single taxon. Micro-algae abundance at seven stations of Hwadang-ri ranged from 1.0 $\times 10^{2}$ to $27,579 \times 10^{2}$ cells $/ 1$ for four seasons. Mean biomass per season was $568 \times 10^{2}$ cells $/ 1$ with 61 taxa.

\section{Composition of species at water surface}

In the whole sampling on January, a total of 31 taxa and 26 taxa were identified at surface layer and basal layer, respectively. The stations B and C were characterized by high phytoplankton biomass. The relative dominant species were Rhizosolenia setigera, Skeletonema costatum, Pseudo-nitzschia pungens, and Pseudo-nitzschia seriata at seven stations.

On April 2013, a total of 42 taxa were identified at surface layer: Bacillariophyceae 27 taxa, Dinophyceae 12 taxa, two Cryptophyceae taxa, and one Eugenophyceae taxon. The station B was characterized by high phytoplankton biomass. The relative dominant species were three diatoms taxa (Chaetoceros danicus, Chaetoceros pseudocrinitus, and Leptocylindrus danicus) at seven stations.

On August, a total of 48 taxa were identified at surface layer: Bacillariophyceae 35 taxa, Dinophyceae 11 taxa, one Cryptophyceae taxon, and one Eugenophyceae taxon. The relative dominant species were seven diatoms taxa (Chaetoceros danicus, Chaetoceros debilis, Chaetoceros didymus, and Skeletonema costatum) at seven stations.

On October, a total of 41 taxa were identified at surface layer: Bacillariophyceae 34 taxa, Dinophyceae 5 taxa, and Cryptophyceae 2 taxa. The station $B$ was characterized by high phytoplankton biomass and station $G$ was lowest. The relative dominant species were nine diatoms taxa (Chaetoceros didymus, Dactyliosolen fragillisimus, Guinardia delicatula, Leptocylindrus danicus, Melosira moniliformis, and Skeletonema costatum) at seven stations.

\section{Composition of species at basal layer}

The phytoplankton community at basal layer on January was also very diverse (Table 2). A total of 53 taxa were identified at surface layer: Bacillariophyceae 36 taxa, Dinophy- 
Table 1. The composition and biomass at water surface

(unit: $\times 100$ cells/1)

\begin{tabular}{|c|c|c|c|c|c|c|}
\hline \multirow{2}{*}{ Species } & \multicolumn{4}{|c|}{ Season } & \multirow{2}{*}{ Total } & \multirow{2}{*}{$\%$} \\
\hline & Jan & Apr & Aug & Oct & & \\
\hline \multicolumn{7}{|l|}{ Chrysophyceae } \\
\hline Dictyocha fibula & 2 & 3 & & 15 & 20 & 0.01 \\
\hline Dictyocha speculum & 5 & 7 & 21 & 19 & 20 & 0.01 \\
\hline Eugenophyceae & & & & & 52 & 0.04 \\
\hline Eutreptiella gymnastica & 10 & 174 & 26 & & & \\
\hline \multicolumn{7}{|l|}{ Bacillariophyceae } \\
\hline Asterionellopsis glacialis & 7 & 24 & 8 & 6 & 45 & 0.03 \\
\hline Bacteriastrum hyalinum & & & & 1042 & 1042 & 0.75 \\
\hline Chaetoceros affinis & & & 38 & 55 & 93 & 0.07 \\
\hline Chaetoceros costatus & & & 394 & & 394 & 0.28 \\
\hline Chaetoceros compressus & & 125 & 11 & & 136 & 0.10 \\
\hline Chaetoceros curvisetus & & & 37 & 44 & 81 & 0.06 \\
\hline Chaetoceros danicus & 28 & 11462 & 14236 & 1123 & 26849 & 19.35 \\
\hline Chaetoceros debilis & & 1624 & 1018 & 273 & 2915 & 2.10 \\
\hline Chaetoceros decipiens & 6 & 55 & 4 & 18 & 83 & 0.06 \\
\hline Chaetoceros didymus & 124 & 4573 & 4121 & 4025 & 12843 & 9.26 \\
\hline Chaetoceros lorenzianus & 105 & 203 & 103 & 1240 & 1651 & 1.19 \\
\hline Chaetoceros mitra & & & 5 & & 5 & 0.00 \\
\hline Chaetoceros pendulus & 63 & 201 & 19 & 65 & 348 & 0.25 \\
\hline Chaetoceros peruvianus & & & 2 & & 2 & 0.00 \\
\hline Chaetoceros pseudocrinitus & & 20146 & 441 & 789 & 21376 & 15.41 \\
\hline Chaetoceros sp. & 21 & 56 & 167 & 54 & 298 & 0.22 \\
\hline Coscinodiscus gigas & 3 & & & 15 & 18 & 0.01 \\
\hline Coscinodiscus wailesii & & & 31 & & 31 & 0.02 \\
\hline Coscinodiscus sp. & 21 & 2 & 14 & 25 & 62 & 0.05 \\
\hline Cylindrotheca closterium & & 3 & 67 & 51 & 121 & 0.09 \\
\hline Dactyliosolen fragillisimus & 217 & 105 & 132 & 27125 & 27579 & 19.88 \\
\hline Dictylum brightwellii & & 15 & 18 & 16 & 49 & 0.04 \\
\hline Eucampia zodiacus & & & & 106 & 106 & 0.08 \\
\hline Guinardia delicatula & 110 & 211 & 22 & 4971 & 5314 & 3.83 \\
\hline Lauderia annulata & & 3 & 6 & 78 & 87 & 0.06 \\
\hline Leptocylindrus danicus & 53 & 10106 & 238 & 2381 & 12778 & 9.21 \\
\hline Leptocylindrus minimus & 132 & & & 77 & 209 & 0.15 \\
\hline Melosira juergensii & & & & 3 & 3 & 0.00 \\
\hline Melosira moniliformis & 72 & & & 2137 & 2209 & 1.59 \\
\hline Navicula sp. & 21 & 2 & 24 & 7 & 54 & 0.04 \\
\hline Nitzschia sp. & 6 & 42 & 27 & 201 & 276 & 0.20 \\
\hline Paralia sulcata & 17 & 31 & 43 & 6 & 97 & 0.07 \\
\hline Pleurosigma angulatum & 20 & 20 & 20 & 108 & 168 & 0.12 \\
\hline Pseudo-nitzschia pungens & 289 & 147 & 744 & 237 & 1417 & 1.02 \\
\hline Pseudo-nitzschia seriata & & & 41 & 305 & 346 & 0.25 \\
\hline Rhizosolenia hebetata & & & 34 & & 34 & 0.03 \\
\hline Rhizosolenia setigera & 1003 & 56 & 25 & 1179 & 2263 & 1.63 \\
\hline Skeletonema costatum & 1032 & 1397 & 2746 & 10136 & 15311 & 11.04 \\
\hline Thalassiosira angulata & 171 & 115 & 113 & & 399 & 0.29 \\
\hline Thalassiosira rotula & 26 & 54 & 77 & 12 & 169 & 0.12 \\
\hline Thalassiosira sp. & 22 & 18 & 16 & 5 & 61 & 0.04 \\
\hline \multicolumn{7}{|l|}{ Dinophyceae } \\
\hline Ceratium furca & & & 21 & 13 & 34 & 0.03 \\
\hline Ceratium fusus & 11 & 425 & 341 & 31 & 808 & 0.58 \\
\hline Ceratium trichoceros & & & 3 & & 3 & 0.00 \\
\hline Dinophysis acuminata & & 5 & 2 & & 7 & 0.01 \\
\hline Gyrodinium fissum & & 6 & 5 & & 11 & 0.01 \\
\hline
\end{tabular}


Table 1. Continued

\begin{tabular}{|c|c|c|c|c|c|c|}
\hline \multirow{2}{*}{ Species } & \multicolumn{4}{|c|}{ Season } & \multirow{2}{*}{ Total } & \multirow{2}{*}{$\%$} \\
\hline & Jan & Apr & Aug & Oct & & \\
\hline Gyrodinium spirale & & 29 & 33 & & 62 & 0.05 \\
\hline Gyrodinium sp. & 1 & & 2 & & 3 & 0.00 \\
\hline Heterocapsa triquetra & & 3 & & 6 & 9 & 0.01 \\
\hline Katodinium glaucum & & 14 & 19 & & 33 & 0.02 \\
\hline Noctiluca scintillans & & 2 & & & 2 & 0.00 \\
\hline Protoperidinium bipes & 3 & & & & 3 & 0.00 \\
\hline Prorocentrum bronchi & & 12 & 7 & & 19 & 0.01 \\
\hline Protoperidinium sp. & & 3 & 1 & 2 & 6 & 0.00 \\
\hline Protoperidinium pellucidum & 11 & 17 & & & 28 & 0.02 \\
\hline Scrippsiella trochoidea & & 33 & 56 & 11 & 100 & 0.07 \\
\hline Torodinium teredo & & 2 & & & 2 & 0.00 \\
\hline Total & 3612 & 51531 & 25579 & 58012 & 138734 & 100 \\
\hline Species No. & 31 & 42 & 48 & 41 & & \\
\hline
\end{tabular}

Table 2. The composition and biomass at basal layer

(unit: cells/l)

\begin{tabular}{|c|c|c|c|c|c|c|}
\hline \multirow{2}{*}{ Species } & \multicolumn{4}{|c|}{ Season } & \multirow{2}{*}{ Total } & \multirow{2}{*}{$\%$} \\
\hline & Jan & Apr & Aug & Oct & & \\
\hline \multicolumn{7}{|l|}{ Chrysophyceae } \\
\hline Dictyocha fibula & & & & 5 & 5 & 0.00 \\
\hline Dictyocha speculum & 14 & 23 & 8 & 11 & 56 & 0.05 \\
\hline \multicolumn{7}{|l|}{ Eugenophyceae } \\
\hline Eutreptiella gymnastica & 17 & 1132 & & & 1149 & 1.00 \\
\hline \multicolumn{7}{|l|}{ Bacillariophyceae } \\
\hline Asterionellopsis glacialis & 26 & 64 & 1125 & 28 & 1243 & 1.08 \\
\hline Bacteriastrum hyalinum & & & 542 & & 542 & 0.47 \\
\hline Chaetoceros affinis & & & 345 & & 345 & 0.30 \\
\hline Chaetoceros costatus & & 341 & 23 & & 364 & 0.32 \\
\hline Chaetoceros compressus & & 48 & & & 48 & 0.04 \\
\hline Chaetoceros curvisetus & & 333 & 55 & & 388 & 0.34 \\
\hline Chaetoceros danicus & 21 & 6115 & 1251 & 27 & 7414 & 6.44 \\
\hline Chaetoceros debilis & & 1568 & 347 & & 1915 & 1.66 \\
\hline Chaetoceros decipiens & 47 & 179 & & 108 & 334 & 0.29 \\
\hline Chaetoceros didymus & 15 & 3458 & 2159 & 44 & 5676 & 4.93 \\
\hline Chaetoceros lorenzianus & 152 & 1103 & 24 & 105 & 1384 & 1.20 \\
\hline Chaetoceros mitra & & & 57 & & 57 & 0.05 \\
\hline Chaetoceros pendulus & 58 & 245 & 2306 & 52 & 2661 & 2.31 \\
\hline Chaetoceros pseudocrinitus & & 1236 & 2476 & 35 & 3747 & 3.26 \\
\hline Chaetoceros sp. & 56 & 159 & 1012 & 269 & 1496 & 1.30 \\
\hline Coscinodiscus gigas & & & & 74 & 74 & 0.06 \\
\hline Coscinodiscus sp. & 63 & & 28 & 31 & 122 & 0.11 \\
\hline Cylindrotheca closterium & & 15 & 194 & 59 & 268 & 0.23 \\
\hline Dactyliosolen fragillisimus & 94 & 301 & 8987 & 23 & 9405 & 8.17 \\
\hline Dictylum brightwellii & & 25 & 215 & 14 & 254 & 0.22 \\
\hline Dictylum pumila & & 21 & 113 & & 134 & 0.12 \\
\hline Eucampia zodiacus & & & 1457 & 88 & 1545 & 1.34 \\
\hline Guinardia delicatula & 103 & 204 & 241 & 12 & 560 & 0.49 \\
\hline Lauderia annulata & & 103 & 161 & & 264 & 0.23 \\
\hline Leptocylindrus danicus & 49 & 15128 & 5346 & 345 & 20868 & 18.13 \\
\hline Melosira moniliformis & 13 & & & & 13 & 0.01 \\
\hline Navicula sp. & 14 & & 248 & 28 & 290 & 0.25 \\
\hline Nitzschia sp. & 25 & 88 & 253 & 11 & 377 & 0.33 \\
\hline Paralia sulcata & 44 & 22 & 105 & 26 & 197 & 0.17 \\
\hline
\end{tabular}


Table 2. Continued

\begin{tabular}{|c|c|c|c|c|c|c|}
\hline \multirow{2}{*}{ Species } & \multicolumn{4}{|c|}{ Season } & \multirow{2}{*}{ Total } & \multirow{2}{*}{$\%$} \\
\hline & Jan & Apr & Aug & Oct & & \\
\hline Pleurosigma angulatum & 55 & 57 & 247 & & 359 & 0.31 \\
\hline Pseudo-nitzschia pungens & 1567 & 125 & 1350 & 52 & 3094 & 2.69 \\
\hline Pseudo-nitzschia seriata & 477 & 16 & 1168 & 27 & 1688 & 1.47 \\
\hline Rhizosolenia setigera & 106 & 17 & 54 & 22 & 199 & 0.17 \\
\hline Skeletonema costatum & 159 & 2045 & 25605 & 82 & 27891 & 24.23 \\
\hline Thalassiosira angulata & 43 & 118 & 397 & 89 & 647 & 0.56 \\
\hline Thalassiosira rotula & 49 & 103 & 402 & 44 & 598 & 0.52 \\
\hline \multicolumn{7}{|l|}{ Dinophyceae } \\
\hline Ceratium furca & & & & 123 & 123 & 0.11 \\
\hline Ceratium fusus & 27 & 31 & 16 & 20 & 94 & 0.08 \\
\hline Dinophysis acuminata & & 547 & 109 & & 656 & 0.57 \\
\hline Gyrodinium fissum & & 14 & & & 14 & 0.01 \\
\hline Gyrodinium spirale & & 61 & & & 61 & 0.05 \\
\hline Heterocapsa triquetra & & 43 & & & 43 & 0.04 \\
\hline Katodinium glaucum & & 114 & & & 114 & 0.10 \\
\hline Protoperidinium bipes & & & 141 & & 141 & 0.12 \\
\hline Prorocentrum bronchi & & 12 & & & 12 & 0.01 \\
\hline Prorocentrum claudicans & & 26 & 1024 & 3 & 1053 & 0.92 \\
\hline Prorocentrum dentatum & & & 26 & & 26 & 0.02 \\
\hline Prorocentrum minimum & 16 & & 14734 & 204 & 14954 & 12.99 \\
\hline Protoperidinium sp. & & 5 & & & 5 & 0.00 \\
\hline Protoperidinium pellucidum & & & & 46 & 46 & 0.04 \\
\hline Scrippsiella trochoidea & & 42 & & 44 & 86 & 0.08 \\
\hline Total & 3310 & 35287 & 74351 & 2151 & 115099 & 100 \\
\hline Species No. & 26 & 40 & 39 & 33 & & \\
\hline
\end{tabular}

ceae 15 taxa, one Cryptophyceae taxon, and one Eugenophyceae taxon. Diatoms and dinoflagellates were the most diverse groups. Centric and pennate diatoms accounted for the highest diversity among of them. Results of $2 \mathrm{~m}, 4 \mathrm{~m}$, $6 \mathrm{~m}$, and $8 \mathrm{~m}$ were not shown in table and they used for water circulation analysis.

On April, a total of 40 taxa were identified at low surface layer: Bacillariophyceae 28 taxa, Dinophyceae 10 taxa, Cryptophyceae and Eugenophyceae, each of one taxon. The station $\mathrm{C}$ was characterized by high phytoplankton biomass. The relative dominant species were two Dinoflagellate taxa (Chaetoceros danicus and Leptocylindrus danicus) at seven stations.

On August, a total of 39 taxa were identified at low layer: Diatoms 32 taxa, Dinoflagellate 6 taxa. The station A was characterized by high phytoplankton biomass. The relative dominant species were three diatoms taxa (Dactyliosolen fragillisimus, Leptocylindrus danicus, and Skeletonema costatum) and one Dinoflagellate taxon (Prorocentrum minimum) at seven stations.

On October, a total of 33 taxa were identified at low layer: Bacillariophyceae 25 taxa, Dinophyceae 6 taxa, and Crypto- phyceae 2 taxa. The station $\mathrm{C}$ was characterized by high phytoplankton biomass and station $\mathrm{G}$ was lowest. Leptocylindrus danicus was the most dominant species at seven stations.

\section{Biomass}

The density and biomass of species demonstrated significant patterns from the near seaside station (Stations B, $\mathrm{C}$, and D) to remote seaside stations (Stations F, G). Winter season was characterized by minimum phytoplankton concentrations and Pseudo-nitzschia pungens was dominant species. During spring season, Leptocylindrus danicus was most abundant $(p<0.01)$ at stations A, C, and F (Table 1). During winter season, Pseudo-nitzschia pungens was most abundant $(p<0.01)$ at stations B and C. Skeletonema costatum was the second dominant species at station A. Rhizosolenia setigera was also abundant at station E.

During spring season, Chaetoceros pseudocrinitus was most abundant at all stations. Leptocylindrus danicus was the second dominant species. Chaetoceros danicus was also abundant at all stations. They were decreased remote seaward $(p<0.01)$.

During summer season, Skeletonema costatum was most abundant at all stations. Prorocentrum minimum was the sec- 
ond dominant species at the stations F and G. Chaetoceros danicus was also abundant at all stations.

During autumn season, Dactyliosolen fragillisimus was most abundant at all stations. Skeletonema costatum was the second dominant species at all stations.

Biomass of species at water surface on January was varied from 200 cells/1 to 103,200 cells/1 (Fig. 2). The station B was shown the highest phytoplankton density among seven stations. Biomass of species at basal layer was 1,400 156,700 cells/l and the station $\mathrm{C}$ was also shown the highest phytoplankton density among seven stations.

Mean biomass of species at water surface and basal layer on April were 1,227 cells/1 and 882 cells/1. Mean biomass of species at water surface and basal layer on August were 533 cells/1 and 188 cells/1. Mean biomass of species at water surface and basal layer on October were 1,415 cells/1 and 65 cells $/ 1$.

\section{Cluster analysis}

Analysis of seasonal variability within the phytoplankton community was performed using the hierarchical clustering using the Jaccard Index of similarity. In the beginning of the year (January), all stations were often more than 60\% of similarity, as shown in Fig. 3. Water surface and basal layer showed a clear distinction excluding Stations $B$ and C (data not shown). Stations A, B, and C formed same clustered (cluster-1). They are mainly consisted of Pseudonitzschia pungens, Rhizosolenia setigera, and Skeletonema costatum which were dominant in cluster-1 on winter. In spring, the plankton community consisted mainly of Chaetoceros pseudoc-
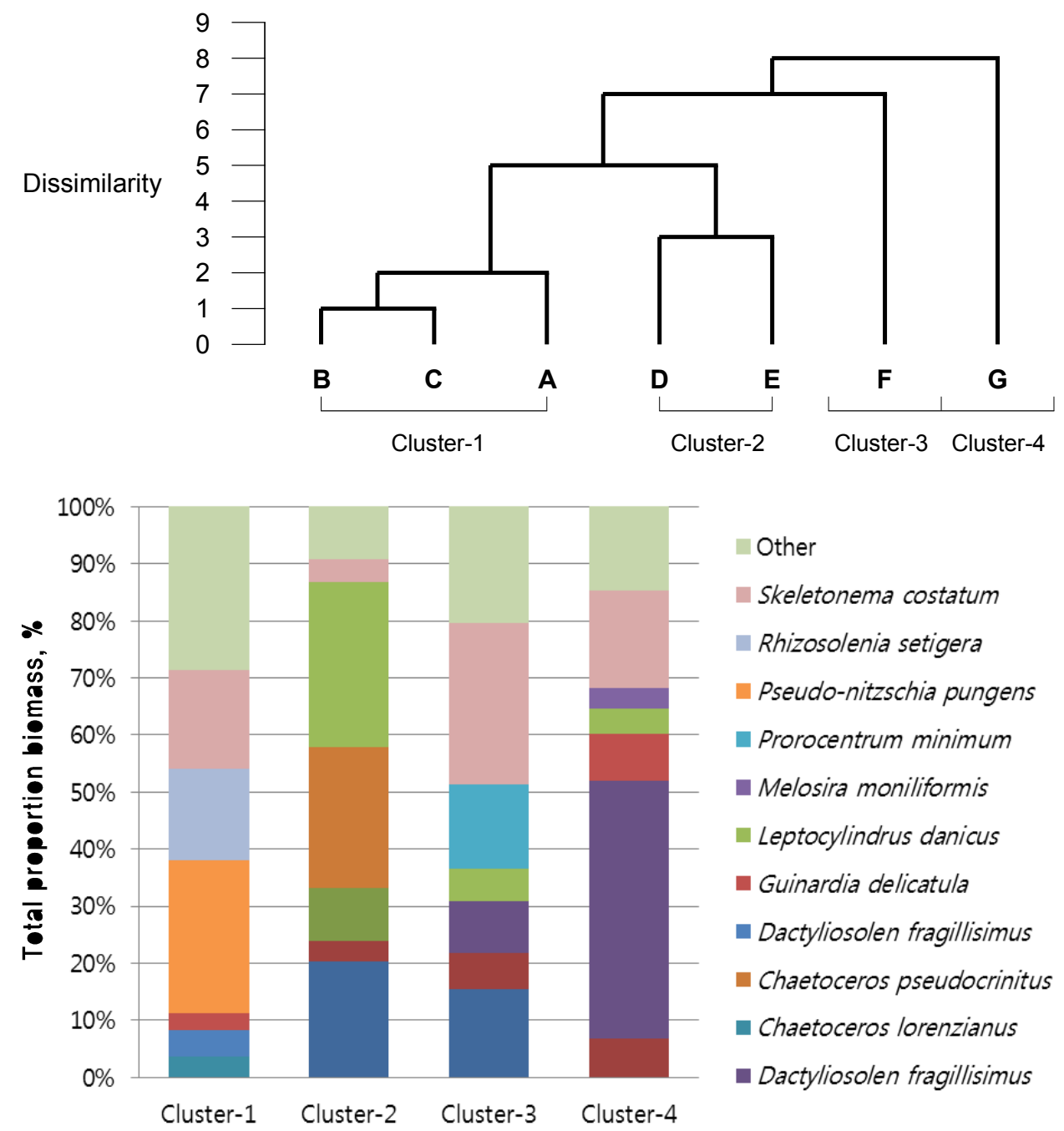

Fig. 2. Spatial variability of phytoplankton community. Upper dendrogram of the cluster analysis based on the dissimilarity among seven stations. Lowe is the compositions of dominant species in different phytoplankton associations within area outlined by the cluster analysis. 


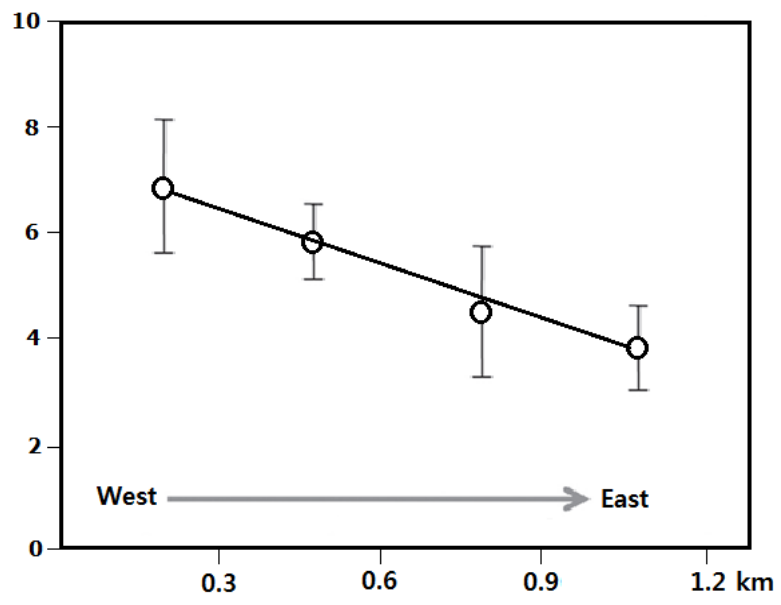

Fig. 3. Simple linear regression in the phytoplankton community along an East-West longitude gradient. The vertical lines represent the SD.

rinitu and Leptocylindrus danicus (Cluster-2). Relative remote stations ( $F$ and $G$ ) were characterized by low similarity between stations or two layers in depths and minimal values of species diversity and community evenness. In late autumn, the phytoplankton community consisted mainly of Skeletonema costatum and Pseudo-nitzschia pungens.

\section{Spatial and temporal variability of phytoplankton}

Water surfaces were shown with the relative individual density or abundance across areas (Table 1 and Table 2). However, Shannon-Weaver indices of diversity of water surfaces were lower than those of basal layers except January (Table 3). In addition, evenness indices of basal layers were higher than those of water surfaces except January.

The station A had high number of species as well as those of both area, B and C (data not shown). Shannon-Weaver index of diversity also varied among the stations and season with 3.023 (October) and 2.255 (August) having higher value than the other stations and season.

Assessments of the seven spatial and four seasonal variability of the structure of the phytoplankton community were presented in Table 4. Although the numbers of species with absolute occurrence were existed in stations and season, mean paired similarity between both the species composition within stations and within seasons were high levels. For the community as a whole, the values of B-diversity were the low (1.125 for water surface within seven stations and 1.481 for basal layer) or common (1.725 for water surface within four seasons and 1.347 for basal layer). They indicated that heterogeneity in species compositions among

Table 3. Biological diversity of phytoplankton in season at Hwadang-ri

\begin{tabular}{|c|c|c|c|c|c|c|c|c|}
\hline \multirow{3}{*}{ Indices } & \multicolumn{8}{|c|}{ Season } \\
\hline & \multicolumn{2}{|c|}{ Jan } & \multicolumn{2}{|c|}{ Apr } & \multicolumn{2}{|c|}{ Aug } & \multicolumn{2}{|c|}{ Oct } \\
\hline & W.S. & B.L. & W.S. & B.L. & W.S. & B.L. & W.S. & B.L. \\
\hline \multicolumn{9}{|c|}{ Diversity } \\
\hline $\mathrm{H}^{\prime}$ & 2.246 & 2.114 & 1.700 & 2.056 & 1.639 & 2.255 & 1.875 & 3.023 \\
\hline N1 & 9.449 & 8.274 & 5.472 & 7.815 & 5.148 & 9.536 & 6.521 & 20.552 \\
\hline N2 & 5.672 & 3.930 & 3.992 & 4.298 & 2.852 & 5.476 & 3.757 & 14.395 \\
\hline \multicolumn{9}{|l|}{ Richness } \\
\hline $\mathrm{R} 1$ & 3.662 & 3.085 & 3.779 & 3.725 & 4.631 & 3.388 & 3.647 & 4.170 \\
\hline $\mathrm{R} 2$ & 0.516 & 0.452 & 0.185 & 0.213 & 0.300 & 0.143 & 0.170 & 0.712 \\
\hline \multicolumn{9}{|c|}{ Evenness } \\
\hline E1 & 0.654 & 0.649 & 0.455 & 0.557 & 0.423 & 0.616 & 0.505 & 0.865 \\
\hline E2 & 0.305 & 0.318 & 0.130 & 0.195 & 0.107 & 0.245 & 0.159 & 0.623 \\
\hline E3 & 0.282 & 0.291 & 0.109 & 0.175 & 0.088 & 0.225 & 0.138 & 0.611 \\
\hline E4 & 0.600 & 0.475 & 0.729 & 0.55 & 0.554 & 0.574 & 0.576 & 0.7 \\
\hline E5 & 0.553 & 0.403 & 0.669 & 0.484 & 0.447 & 0.524 & 0.499 & 0.685 \\
\hline
\end{tabular}

Table 4. Space-time variability of the phytoplankton community structure

\begin{tabular}{lcccc}
\hline \multirow{2}{*}{ Attributes of community structure } & Spatial variability & (7 stations) & \multicolumn{2}{c}{ Temporal variability $(4$ seasons $)$} \\
\cline { 2 - 5 } & Water surface & Basal Layer & Water surface & Basal Layer \\
\hline Mean number of species per sample & 28 & 25 & 41 & 35 \\
Number of species with absolute occurrence & 7 & 5 & 11 & 16 \\
Number of samples containing all species & 12 & 8 & 22 & 19 \\
Occurrence index (B-diversity) & 1.481 & 1.125 & 1.725 & 1.347 \\
\hline
\end{tabular}


the replicates were not high. The parameters paired similarity between season and stations testified (Table 5). There were high taxonomic homogeneity of the phytoplankton community in between four seasons and similar trends in seasonal development of phytoplankton at depths of same stations. However, size distribution of abundance and biomass showed a statistically significant west-east different $(p$ $<0.05$, Table 6).

In order to assess macro-scale spatial variability of the phytoplankton community at Hwadang-ri, I analyzed distributions of species richness and diversity of large taxonomic groups as well as phytoplankton composition along a longitudinal gradient. Figure 3 showed the biomass plotted against longitude. Margalef's index gradually decreased from west to east. This trend conformed to a linear regression model, which described $85 \%$ of the spatial variability for mean species biomass $\left(r^{2}=0.67\right)$.

Phytoplankton composition from western areas near the Hwadang-ri was more diverse than that of eastern areas. This decreasing trend was supported mainly by an increase of phytoplankton diversity. The mean number of species within the western waters was 55 taxa and eastern was 44 . The portion of dinoflagellates in the phytoplankton decreased exponentially along the west-east gradient (Fig. 4). The diatom/dinoflagellate ratio was equal to 0.768 within western waters (Stations A, B, C); whereas it was reduced to 0.894 in middle waters, and even further to 1.044 in open waters.

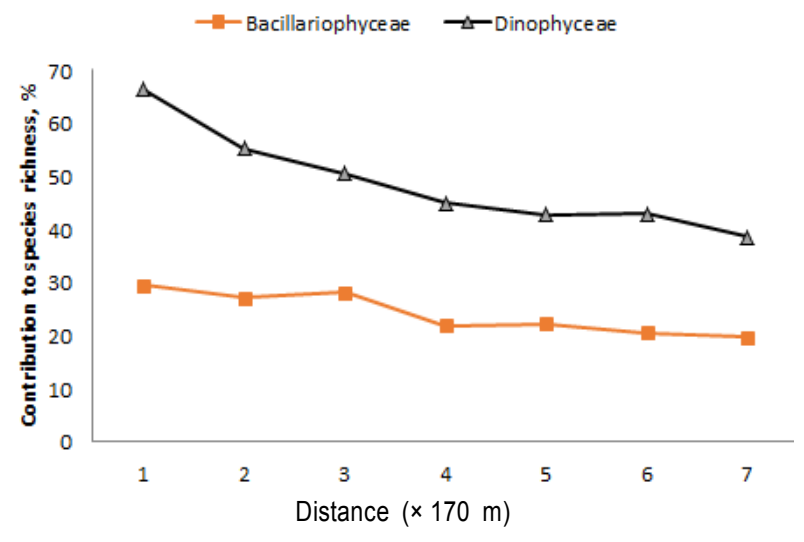

Fig. 4. Percentage contribution of phytoplankton groups to the total species richness plotted against geographical distances.

\section{Discussion}

Diatoms dominated phytoplankton abundance numerically as well as in biomass, accounting for $98.98 \%$ of the latter depending on season. Phytoplankton concentrations, which were obtained in this study, are within the range of those reported previously [2]. Both the spatial and temporal components contributed to the variability of the phytoplankton community at Hwadang-ri in Goseng-gun. During the winter months, the western area was characterized by the lowest concentrations of phytoplankton. It was strongly correlated with temperature from cold river introduction of inland, whereas the highest phytoplankton concentrations were observed in open waters. The minimum diversity level

Table 5. Test of homogeneity of the phytoplankton community between the four seasons at Hwadang-ri

\begin{tabular}{ccccc}
\hline Month & January & April & August & October \\
\hline January & - & $p>0.05$ & $p>0.05$ & $p>0.05$ \\
April & $0.159(32)$ & - & $p>0.05$ & $p>0.05$ \\
August & $0.794(45)$ & $0.211(37)$ & - & $p>0.05$ \\
October & $1.927(44)$ & $0.223(39)$ & $0.084(44)$ & - \\
\hline
\end{tabular}

Upper diagonal is the degree of significant for $p$ value and low diagonal is $t$ value. Parenthesis is the degree of freedom.

Table 6. Test of homogeneity of the phytoplankton community between the seven stations at Hwadang-ri

\begin{tabular}{cccccccc}
\hline Station & $\mathrm{A}$ & $\mathrm{B}$ & $\mathrm{C}$ & $\mathrm{D}$ & $\mathrm{E}$ & $\mathrm{F}$ & $\mathrm{G}$ \\
\hline $\mathrm{A}$ & - & $p>0.05$ & $p>0.05$ & $p<0.01$ & $p<0.01$ & $p<0.01$ & $p<0.01$ \\
B & -0.038 & - & $p>0.05$ & $p<0.05$ & $p<0.05$ & $p<0.01$ & $p<0.01$ \\
C & 0.210 & 0.222 & - & $p>0.05$ & $p<0.05$ & $p<0.01$ & $p<0.01$ \\
D & 3.149 & 2.320 & 1.815 & - & $p>0.05$ & $p<0.01$ & $p<0.01$ \\
E & 3.237 & 2.531 & 2.536 & 1.962 & - & $p>0.05$ & $p<0.05$ \\
F & 3.547 & 3.015 & 2.731 & 2.611 & 1.685 & - & $p>0.05$ \\
G & 3.268 & 2.854 & 2.711 & 2.477 & 1.608 & -0.084 & - \\
\hline
\end{tabular}

Upper diagonal is the degree of significant for $p$ value and low diagonal is $t$ value. 
was associated with the winter months, whereas the maximum was in autumn (October). Phytoplankton species richness gradually increased eastward, with the lowest richness recorded in the waters closest to the coastal-side (Stations A, B, C) and the highest towards the eastern waters (Stations F, G). These east-west differences in the phytoplankton community have been reported for the Pacific $[4,10,16]$. This east-west difference in the phytoplankton community may reflect heterogeneity in the grazing pressure of the zooplankton community $[9,19]$ or temperature and magnitude of the spring phytoplankton bloom [21].

Most of the time, marine waters are characteristically blue or green and reasonably clear. In the temperate waters of the northern latitudes, water is seldom as clear as seen in tropical areas, where visibility can exceed 50-75 feet [22]. In temperate waters, the limits of visibility or murkiness are usually the result of algae in the water. However, in some unusual cases, a single microalgal species can increase in abundance until they dominate the microscopic plant community and reach such high concentrations that they discolor the water with their pigments, these "blooms" of algae are often referred to as a "Red tide". Although referred to as Red tides, blooms are not only red, but can be brown, yellow, green, or milky in color [7]. Adverse effects can likewise occur when algal cell concentrations are low and these cells are filtered from the water by shellfish such as clams, mussels, oysters, scallops, or small fish. Many animals at higher levels of the marine food chain are impacted by harmful algal blooms. Toxins can be transferred through successive levels of the food chain, sometimes having lethal effects.

Euglenophyceae (Eutreptiella gymnastica) was found in the four stations on January and the all stations on April in this study (Table 1 and Table 2) to be the major bloom formers [7]. Chaetoceros curvisetus, Leptocylindrus danicus, Skeletonema costatum, Cylindrotheca closterium, Pseudo-nitzschia pungens, Navicula spp. of Bacillariophyceae were also found in most stations and seasons to be the major bloom formers [7]. Ceratium furca, Ceratium fusus, Gyrodinium fissum, Heterocapsa triquetra, Prorocentrum dentatum, Prorocentrum minimum, Scrippsiella trochoidea of Dinophyceae were found to be the major bloom formers.

We expect that this work may provide valuable information of interest to later ecological studies. Definitive identification of the principal phytoplankton species assumes greater importance also at the light of the potentially serious and harmful effects associated with bloom events.

\section{Acknowledgement}

This work was supported by Dong-eui University Grant (2014AA472).

\section{References}

1. Almandoz, G. O., Hernando, M. P., Ferreyra, G. A., Schloss, I. R. and Ferrario, M. E. 2011. Seasonal phytoplankton dynamics in extreme southern South America (Beagle Channel, Argentina). J Sea Res 66, $47-57$.

2. Al-Zaidan, A. S. Y., Kennedy, H., Jones, D. A. and Al-Mohanna, S. Y. 2006. Role of microbial mats in Sulaibikht Bay (Kuwait) mudflat food webs: evidence from $\delta 13 \mathrm{C}$ analysis. Marine Ecology Progress Series 308, 27 - 36.

3. Falkowski, P. G., Laws, E. A., Barber, R. T. and Murray, J. W. 2003. Phytoplankton and their role in primary, new, and export production, pp. 99-121. In: Fasham, M. J. R. (ed.), Ocean biogeochemistry: This Role of the Ocean Carbon Cycle in Global Change Springer, Berlin, Heidelberg, NY.

4. Harrison, P. J., Boyd, P. W., Varela, D. E., Takeda, S., Shiomoto, A. and Odate, T. 1999. Comparison of factors controlling phytoplankton productivity in the NE and NW subarctic Pacific gyres. Prog Oceanogr 43, 205 - 234.

5. Hill, M. O. 1973. Diversity and evenness: a unifying notation and its consequences. Ecology 54, 423-432.

6. IBM Corp. Released 2012. IBM SPSS Statistics for Windows, Version 21.0. Armonk, NY: IBM Corp.

7. Lee, D. K. 2008. Cochlodinium polykrikides blooms and ecophysical conditions in the South Sea of Korea. Harmful Algae 7, 318-323.

8. Lee, S. G., Kim, H. G., Bae, H. M., Kang, Y. S., Jeong, C. S., Lee, C. K., Kim, S. Y., Kim, C. S., Lim, W. A. and Cho, U. S. 2002. Handbook of Harmful Marine Algal Blooms in Korean Waters, pp. 172, National Fisheries Research and Development Institute, Republic of Korea, Seoul.

9. Mackas, D. L. and Tsuda. A. 1999. Mesozooplankton in the eastern and western subarctic Pacific: community structure, seasonal life histories, and interannual variability. Prog Oceanogr 43, 335 - 363.

10. Magurran, A. E. 1988. Ecological diversity and its measurement, pp. 192, Princeton Univ. Press, Cambridge, USA.

11. Matsuno, K. and Yamaguchi, A. 2010. Abundance and biomass of mesozooplankton along north-south transects $\left(165^{\circ} \mathrm{E}\right.$ and $\left.165^{\circ} \mathrm{W}\right)$ in summer in the North Pacific: an analysis with an optical plankton counter. Plankton Benthos Res 5, 123-130.

12. McWilliams, J. C. 1996. Modeling the oceanic general circulation. Annu Rev Fluid Mech 28, 215-248.

13. Merritt, R. W. and Cummins, K. W. 1996. An Introduction to the Aquatic Insects of North America, pp. 862, 3rd ed., Kendall/Hunt., Dubuque, Iowa. 
14. Pielou, E. C. 1966. The measurement of diversity in different types of biological collection. J Theoret Biol 13, 131-144.

15. Shannon, C. E. and Weaver, W. 1963. The Measurement Theory of Communication, pp. 1-117, Univ. of Illinois Press, Urbana, USA.

16. Shiomoto, A. and Hashimoto, S. 2000. Comparison of east and west chlorophyll $a$ standing stock and oceanic habitat along the transition domain of the North Pacific. J Plankton Res 22, 1-14.

17. Sournia, A. 1978. Phytoplankton manual. In Monographs on Oceanographic Methodology 6, pp. 337, UNESCO, Paris.

18. Stanca, E., Roselli, L. Cellamare, M. and Basset, A. 2013. Phytoplankton composition in the coastal Magnetic Island lagoon, Western Pacific Ocean (Australia) TWB, Transit. Waters Bull 7, 145-158.
19. Takahashi, K., Kuwata, A., Saito, H. and Ide, K. 2008. Grazing impact of the copepod community in the Oyashio region of the western subarctic Pacific Ocean. Prog Oceanogr $78,222-240$.

20. Tomas, C. R. 1997. Identifying marine phytoplankton. Academic press, pp. 858, Harcourt Brace and Company, Toronto

21. Tsuda, A., Saito, H. and Kasai, H. 2004. Life histories of Eucalanus bungii and Neocalanus cristatus (Copepoda: Calanoida) in the western subarctic Pacific Ocean. Fish Oceanogr 13, 10-20.

22. Verlencar, X. N. 2004. Phytoplankton Identification Manual, pp. 1-40, National Institute of Oceanography, Dona Paula, India.

23. Wiederholm, T. 1983. Chironomidae of the Holarctic region Keys and Diagnoses. Part 1-Larvae, pp. 457, Entomologica Scandinavica Supplement, Motala.

\section{초록 : 고성군 화당리 연안에서 식물플랑크톤의 계절 및 지점별 조성 변화}

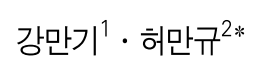

(동의대학교 자연 · 생활과학대학 데이터정보학과, ${ }^{2}$ 동의대학교 자연 - 생활과학대학 분자생물학과)

본 연구는 2013년 고성군 화당리에 있는 7개 지점에 대한 식물 플랑크톤의 공간적 분포, 계절적 분포, 표층과 심층의 깊이에 따른 빈도에 대해 기술한 것이다. 화당리에서 식물 플랑크톤 군집은 3 강 60 분류군으로 다양하였다. 규조강(Bacillariophyceae)은 41분류군으로 가장 높은 다양성을 나타내었으며 그 다음으로는 와편모강(Dinophyceae)으로 16분류군이었고, 황금색조식물강(Cryptophyceae)이 2분류군, 유글레나식물강(Eugenophyceae)이 1분류 군이었다. 표층은 비교적 높은 밀도와 풍부도를 유지하고 있었다. 그런데 Shannon-Weaver의 다양도 지수는 1월 을 제외하고는 표층보다 저층에서 더 높았다. 또 균등도 지수도 1 월을 제외하고는 표층보다 저층에서 더 높았다. 전체 군집에 대해 $\beta$-다양도는 낮거나(7개 정점의 공간적 표층은 1.125 , 저층은 1.481$)$ 보통 (7개 정점의 시간적 표층 은 1.725 , 저층은 1.347 )이었다. 계절에 따라서는 식물 플랑크톤의 군집 간에 분류학적 동질성이 있었다. 깊이에 대해서도 역시 동질성을 나타내었다. 그러나 풍부도의 분포와 생체량은 동-서 방향 구배가 유의한 차이를 나타내 었다. 